\title{
Low-field paramagnetic resonance imaging of tumor oxygenation and glycolytic activity in mice
}

\author{
Shingo Matsumoto, ${ }^{1}$ Fuminori Hyodo, ${ }^{1}$ Sankaran Subramanian, ${ }^{1}$ \\ Nallathamby Devasahayam,, Jeeva Munasinghe,,2 Emi Hyodo,1 Chandramouli Gadisetti, ${ }^{3}$ \\ John A. Cook, ${ }^{1}$ James B. Mitchell, ${ }^{1}$ and Murali C. Krishna ${ }^{1}$ \\ ${ }^{1}$ Radiation Biology Branch, Center for Cancer Research, National Cancer Institute, ${ }^{2}$ National Institute of Neurological Disorder and Stroke, and \\ ${ }^{3}$ Radiation Oncology Branch, Center for Cancer Research, National Cancer Institute, NIH, Bethesda, Maryland, USA.
}

\begin{abstract}
A priori knowledge of spatial and temporal changes in partial pressure of oxygen (oxygenation; $\mathrm{PO}_{2}$ ) in solid tumors, a key prognostic factor in cancer treatment outcome, could greatly improve treatment planning in radiotherapy and chemotherapy. Pulsed electron paramagnetic resonance imaging (EPRI) provides quantitative 3D maps of tissue $\mathrm{PO}_{2}$ in living objects. In this study, we implemented an EPRI set-up that could acquire $\mathrm{pO}_{2}$ maps in almost real time for $2 \mathrm{D}$ and in minutes for $3 \mathrm{D}$. We also designed a combined EPRI and MRI system that enabled generation of $\mathrm{pO}_{2}$ maps with anatomic guidance. Using EPRI and an air/carbogen $(95 \%$ $\mathrm{O}_{2}$ plus $5 \% \mathrm{CO}_{2}$ ) breathing cycle, we visualized perfusion-limited hypoxia in murine tumors. The relationship between tumor blood perfusion and $\mathrm{pO}_{2}$ status was examined, and it was found that significant hypoxia existed even in regions that exhibited blood flow. In addition, high levels of lactate were identified even in normoxic tumor regions, suggesting the predominance of aerobic glycolysis in murine tumors. This report presents a rapid, noninvasive method to obtain quantitative maps of $\mathrm{pO}_{2}$ in tumors, reported with anatomy, with precision. In addition, this method may also be useful for studying the relationship between $\mathrm{pO}_{2}$ status and tumor-specific phenotypes such as aerobic glycolysis.
\end{abstract}

\section{Introduction}

By the time a tumor grows to a detectable size, it exhibits significant heterogeneity in critical metabolites such as oxygen, glucose, growth factors, and other nutrients (1). In normal tissues, such gradients exist approximately $400 \mu \mathrm{m}$ from blood supply whereas in tumors these gradients exist within approximately $200 \mu \mathrm{m}(2)$. Tumors exhibit high levels of glucose uptake and lactate production, reflecting a shift to aerobic glycolysis as a result of activation of oncogenes or loss of tumor suppressor genes $(1,3-5)$. This leads to decreased mitochondrial oxygen consumption, which may be an adaptive response to low oxygen conditions, allowing tumor cells to spare scarce oxygen to allow other critical cellular processes dependent on oxygen to proceed (6).

Microenvironmental changes occur as a result of inefficient vascular function within a tumor (7). Tumors become hypoxic because new blood vessels are aberrant and support poor flow patterns (8). Although tumors induce angiogenesis, the blood vessels that form are structurally and functionally abnormal, resulting in marked regional heterogeneity in tumor perfusion. As a result, tumor progression is paradoxically associated with both angiogenesis and hypoxia (9). Longitudinal oxygen gradients in tumors result from lack of redundant arteriolar network leading to diffusion-limited hypoxia (10). Fluctuations in red blood cell flux can cause intravascu-

Nonstandard abbreviations used: ADC, apparent diffusion coefficient; EPR, electron paramagnetic resonance; EPRI, EPR imaging; FOV, field of view; IMRT, intensitymodulated radiation therapy; MRS, magnetic resonance spectroscopy; $\mathrm{pO}_{2}$, partial pressure of oxygen (oxygenation); RF, radio frequency; ROI, region of interest; SCC, squamous cell carcinoma; TAM, triarylmethyl radical; TE, echo time; TR, repetition time; USPIO, ultrasmall superparamagnetic iron oxide.

Conflict of interest: The authors have declared that no conflict of interest exists. Citation for this article: J. Clin. Invest. 118:1965-1973 (2008). doi:10.1172/JCI34928. lar oxygen to vary, causing hypoxia-reoxygenation (8). Instabilities of red blood cell flux may actually be the norm within tumors, leading to widespread instability in partial pressure of oxygen (oxygenation; $\mathrm{pO}_{2}$ ). Thus, vascular stasis may not be the only process underlying transient hypoxia. Tumors classified as radiobiologically hypoxic $\left(\mathrm{pO}_{2}<10 \mathrm{mmHg}\right)$ have been associated with poor treatment outcome (11-13). Hypoxia has a profound impact on tumor behavior and efforts that use hypoxia to predict tumor treatment response are gaining importance $(14,15)$. A priori knowledge of hypoxia is of significant value in treatment planning, such as the use of hypoxic cytotoxins and appropriate staging of radiotherapy (13). Therefore, robust techniques that are capable of monitoring tissue oxygen status in live objects noninvasively and quantitatively will be useful in preclinical research and eventually in clinical use in oncology (16).

Several methods exist to monitor tissue $\mathrm{pO}_{2}$ in living subjects (16). Oxygen-sensing electrodes have thus far provided the most data in human tumors $(14,17,18)$. In this procedure, a fine needle electrode is inserted into accessible tumor sites and the $\mathrm{pO}_{2}$ is measured at several positions along $2-3$ specific tracks. While the electrode measurements of $\mathrm{pO}_{2}$ are quantitative, it is by nature an invasive procedure limiting its utility to only tumor sites that are readily accessible (19). The $\mathrm{pO}_{2}$ information from electrodes is limited to tissue along the needle track in spite of heterogeneous tumor $\mathrm{pO}_{2}$ patterns along all directions (15). Tumor $\mathrm{pO}_{2}$ may also be estimated using nitroheterocycle drugs, which avidly bind hypoxic cells after bioreductive activation and quantify either ex vivo, with biopsies using immunohistochemical analyses, or noninvasively, with positron emission tomography using ${ }^{18} \mathrm{~F}$-labeled nitroheterocycle drugs (20-22). However, the dependence on specific enzymatic reactions necessary to activate agent binding to hypoxic cells makes precise quantitation by these techniques difficult. From a recent summary 


\section{A Pulsed EPRI oxygen imager}
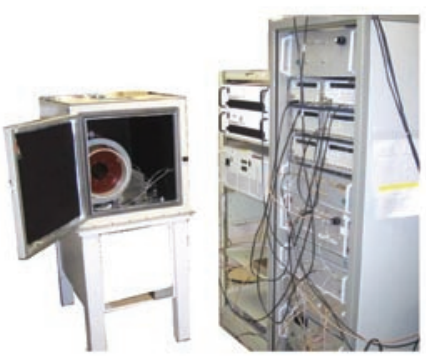

B

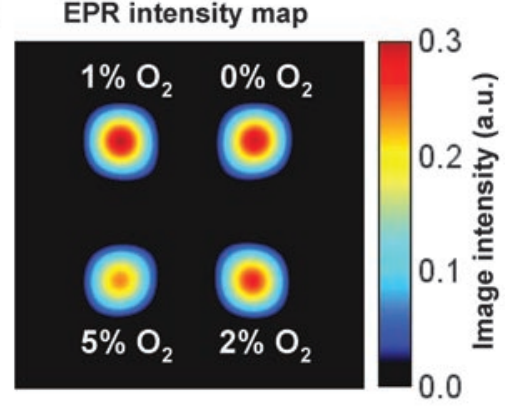

Line width calculation

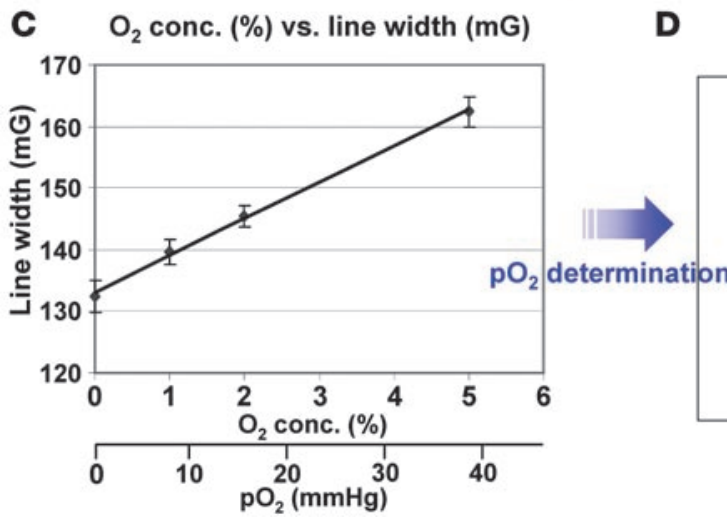

\section{Figure 1}

The pulsed EPR system. (A) EPRI spectrometer used for $\mathrm{pO}_{2}$ imaging. Oxygen imaging is carried out using the $300 \mathrm{MHz}$ pulsed EPR scanner operating at $10 \mathrm{mT}$, having 5 ns time resolution for signal detection. (B) A 4D (3D spatial and 1D spectral) EPR image was obtained after infusion of the oxygen sensitive probe TAM equilibrated at different levels of oxygen as indicated in the figure. Spatially resolved EPR line width of TAM can be extracted from the EPR signal intensity image, which has linear relationship with partial pressure of oxygen (C), and consequently, an absolute $\mathrm{pO}_{2}$ map (D) can be obtained with anatomical overlay by sequential MRI scan of the same object. of the methods being developed/evaluated for clinical use (16), desirable features of $\mathrm{pO}_{2}$ monitoring technique are that (a) the technique is noninvasive and capable of examining sites deep in the human body; (b) quantitative $\mathrm{PO}_{2}$ can be determined with precision in the $\mathrm{PO}_{2}$ range of $0-20 \mathrm{mmHg}$; (c) the technique is capable of providing longitudinal measurements, anatomically coregistered $\mathrm{pO}_{2}$ maps in $3 \mathrm{D}$, with useful spatial and temporal resolution.

Electron paramagnetic resonance (EPR) is a spectroscopic technique similar to nuclear magnetic resonance. EPR detects paramagnetic species that have unpaired electrons such as transition metal complexes and free radicals. With the recent availability of triarylmethyl radical (TAM) probes as in vivo compatible paramagnetic tracers (23), EPR imaging (EPRI) is being explored for mapping tissue oxygen in live animals (24-26). The collisional interaction between TAM and dissolved paramagnetic oxygen leads to a broadening of the spectral line width of TAM. The EPR spectral broadening of TAM is linear with oxygen concentration, providing quantitative capability of EPR in determining tissue $\mathrm{pO}_{2}(27)$. Furthermore, using magnetic field gradients, as in MRI, the spatial distribution of the TAM tracer can be obtained in a living subject (28). By extracting the $\mathrm{pO}_{2}$-dependent EPR line widths, a $\mathrm{pO}_{2}$ map within the body can be generated (25).

While images from EPRI provide maps of $\mathrm{PO}_{2}$, they lack the anatomic detail provided by MRI scans. We therefore designed a combined EPRI plus MRI system, operating at a common frequency of $300 \mathrm{MHz}$ in both modalities, with the corresponding magnetic fields at $10 \mathrm{mT}$ (EPRI) and $7 \mathrm{~T}$ (MRI). Sequential scans with the 2 modalities employing a common resonator enables $\mathrm{pO}_{2}$ maps with anatomic guidance. Also, additional information gathered from MRI such as blood flow, blood volume, and water apparent diffusion coefficients (ADCs) aids in a more complete understanding of tumor physiology. In this report, we describe for the first time to our knowledge, the implementation and performance of pulsed EPR, also named Time Domain EPR, oxygen imaging of a preclinical animal model that can acquire $\mathrm{pO}_{2}$ maps in almost real time for $2 \mathrm{D}$ and in minutes for $3 \mathrm{D}$. Further, it was possible for what we believe to be the first time to choose regions of interest (ROIs) based on $\mathrm{PO}_{2}$ status and examine, using magnetic resonance spectroscopy (MRS), the levels of metabolites such as lactate, choline, etc., which may reflect the glycolytic states in the same regions. The results from this study show that (a) it is possible using EPR and air/carbogen cycling in the inspired gas to obtain spatial information pertaining to perfusion-limited hypoxia in tumors in a living animal. (b) The relationship between blood volume, blood flow, and $\mathrm{pO}_{2}$ status from the tumors can be examined; there appear to be situations where substantial hypoxia may exist even in regions exhibiting blood flow. (c) High levels of lactate indicative of glycolysis, even in normoxic regions of tumors, were identified using sequential EPRI and MRS. This is a first report to our knowledge to simultaneously provide $\mathrm{pO}_{2}$ levels and lactate levels in specific regions of tumor, visualizing the predominance of aerobic glycolysis in normoxic regions of tumors in intact animals.

\section{Results}

The pulsed EPR system used in this study (Figure 1A) has been designed to attain a time resolution of $5 \mathrm{~ns}$, which allows tracking of the $\mathrm{pO}_{2}$-dependent spin dynamics of paramagnetic tracers (29). A phantom object with 4 tubes of aqueous solutions containing $3 \mathrm{mM}$ of the oxygen sensitive paramagnetic tracer TAM equilibrated with $0 \%, 1 \%, 2 \%$, and $5 \%$ oxygen was used to test $\mathrm{pO}_{2}$ imaging capabilities by EPRI (Figure 1B). From the EPR image, the spectral line widths were extracted from the ROI drawn, 


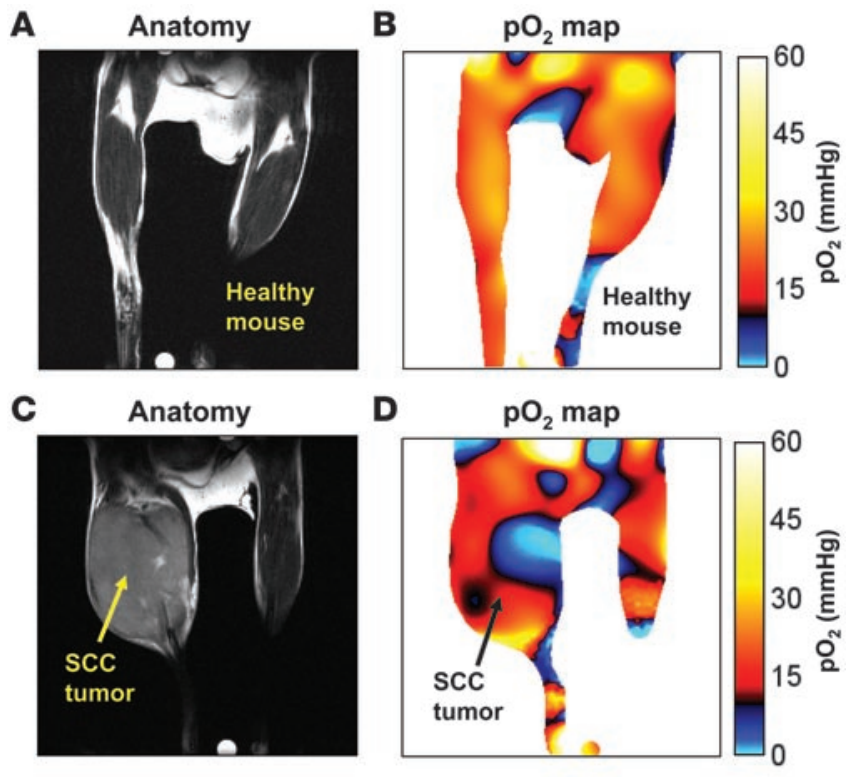

E

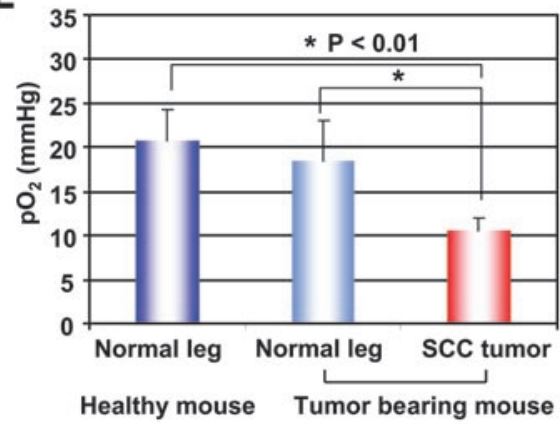

encompassing each of the 4 tubes, and the mean line width values were plotted against oxygen concentration (Figure 1C). The linear relationship between the line width and $\mathrm{pO}_{2}$, and the capability to discriminate differences of 3-4 $\mathrm{mmHg}$ from the EPRI experiments, was evident from the experimental results. Sequentially, the proton MRI image of the same phantom was obtained and overlaid on the $\mathrm{pO}_{2}$ image, finally yielding the anatomically coregistered $\mathrm{pO}_{2}$ image (Figure 1D).

The use of radio frequency (RF) at $300 \mathrm{MHz}$ for EPR permits $\mathrm{PO}_{2}$ imaging deep in the tissue of a living object as with MRI. The tail vein of a healthy mouse was cannulated to permit administration of TAM as a tracer for EPRI. The anatomic image from MRI (Figure $2 \mathrm{~A}$ ) from the lower body of a healthy mouse and corresponding $\mathrm{PO}_{2}$ map from EPRI (Figure 2B) show that the normal muscle tissue is homogeneously well oxygenated $(20.8 \pm 3.3 \mathrm{mmHg} ; n=6)$. The ability of pulsed EPRI to distinguish a 3- to 4-mmHg $\mathrm{pO}_{2}$ difference and its applicability to living objects makes it possible to provide unique and clinically useful information on tumor hypoxia. Figure $2 \mathrm{C}$ shows the anatomic image from MRI of a squamous cell carcinoma (SCC) tumor-bearing mouse (implanted on the right hind leg), and Figure 2D shows the corresponding $\mathrm{PO}_{2}$ map from EPRI. Previous studies showed that the SCC tumor at a size of $1 \mathrm{~cm}^{3}$ exhibits hypoxia in a significant fraction $(25,30)$. There was no significant difference in $\mathrm{pO}_{2}$ between normal muscle tissues with or without tumor as opposed to significant lower $\mathrm{pO}_{2}$ level $(10.5 \pm 3.6 \mathrm{mmHg}$; $n=6 ; P<0.01$ ) in tumor region (Figure $2 \mathrm{E}$ ).

\section{Figure 2}

EPR oxygen imaging of normal muscle in live mice. EPRI method allows the $\mathrm{pO}_{2}$ map from deep in tissue of healthy mouse to be obtained. The anatomic image from MRI (A) of the lower body of a healthy mouse (without tumor bearing) and corresponding $\mathrm{pO}_{2}$ map from EPRI (B) showed that the normal muscle region had relatively homogeneous $\mathrm{pO}_{2}$ distribution. The anatomic image (C) and $\mathrm{pO}_{2}$ image (D) were also obtained from the contra-lateral normal leg of a SCC tumor-bearing mouse, and compared with that of a healthy mouse. (E) There was no significant difference in $\mathrm{pO}_{2}$ between normal muscle tissues with or without tumor bearing as opposed to the significant, lower $\mathrm{pO}_{2}$ in tumor region of the $\mathrm{SCC}$ mouse.

Anatomic imaging using MRI has improved substantially with the availability of scanners with increasing magnetic field strength. The operating RF of MRI at $7 \mathrm{~T}$ being $300 \mathrm{MHz}$ offers opportunities for a common RF chain for both MRI (7 T) and EPRI (10 mT) such that an object can be sequentially scanned for anatomy with MRI and mapped for $\mathrm{pO}_{2}$ by EPRI using the same resonator/gantry. This capability permits reliable coregistration of tissue oxygen maps from EPRI with the anatomic images from MRI without the need to move the object within the resonator/gantry between the 2 scan procedures. Based on this concept, the capability of assessing radiobiologically relevant hypoxic regions in tumors by the pulsed EPRI, anatomic imaging by MRI, and additional physiologic studies using blood pool contrast agents was examined in an experimental model of tumor-bearing mouse. The tumor-bearing mouse was placed in the resonator such that the field of view (FOV) spanned the 2 legs, with the torso placed outside on a platform (Figure 3A). Two tubes containing TAM in water (fiducials) were also included in the FOV so that they could be detected by both the imaging modalities. Figure $3 \mathrm{~B}$ shows the proton transverse relaxation time-weighted ( $\mathrm{T}_{2}$-weighted) MRI anatomic images of the 2 legs in the axial and coronal profiles ( $\mathrm{T}_{2}$ is the proton spin-spin relaxation time). The tumor-bearing leg can be clearly visualized, from which the ROIs were chosen. (EPRI studies were conducted prior to MRI, so that there was no influence of the contrast agents used in MRI on EPR spectral properties of TAM.) The $\mathrm{PO}_{2}$ maps (axial and coronal) obtained from EPRI in the same FOV of the mouse when breathing air are shown in Figure $3 \mathrm{C}$. From the $\mathrm{pO}_{2}$ maps, it can be seen that significant regions of the tumor were hypoxic. Figure $3 \mathrm{D}$ shows the images from the same mouse when the breathing gas that was switched to carbogen $\left(95 \% \mathrm{O}_{2}\right.$ plus $\left.5 \% \mathrm{CO}_{2}\right)$. The $\mathrm{pO}_{2}$ maps from EPRI show that under these conditions, a significant part of the tumor exhibited increase in oxygen as shown in the frequency histogram (Figure 3E). After the EPRI studies, the resonator containing the mouse was moved to the gantry along with the breathing cone and i.v. lines and placed in the 7 T MRI magnet. After acquiring anatomic images (Figure 3B), ultrasmall superparamagnetic iron oxide (USPIO) solution was injected through the cannula in the tail vein. USPIO, which is a $T_{2}$ contrast agent restricted to vasculature, decreases the image intensity from the blood vessels (31). From the difference image (image before contrast agent administration - image after contrast agent administration), absolute blood volume can be calculated (Figure 3F). The blood volume image, when compared with the corresponding anatomic image (Figure $3 \mathrm{~B}$ ) and $\mathrm{pO}_{2}$ image (Figure 3, C and D) supported the following conclusions: (a) the SCC tumor contained regions of heterogeneous blood volume; (b) even in regions in which there was considerable blood 
A
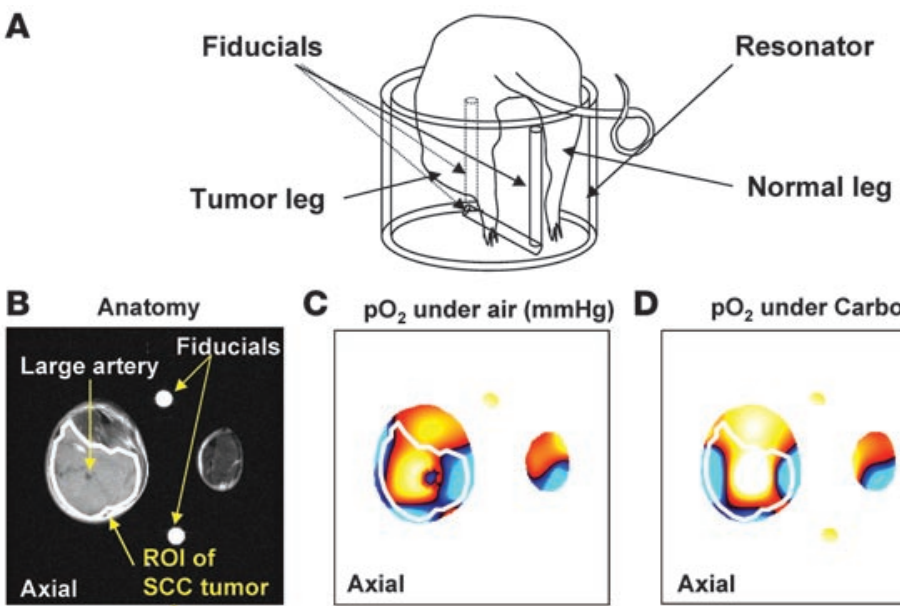

C $\mathrm{pO}_{2}$ under air $(\mathrm{mmHg})$

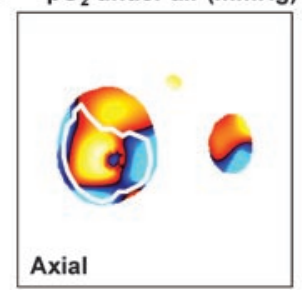

D $\mathrm{pO}_{2}$ under Carbogen
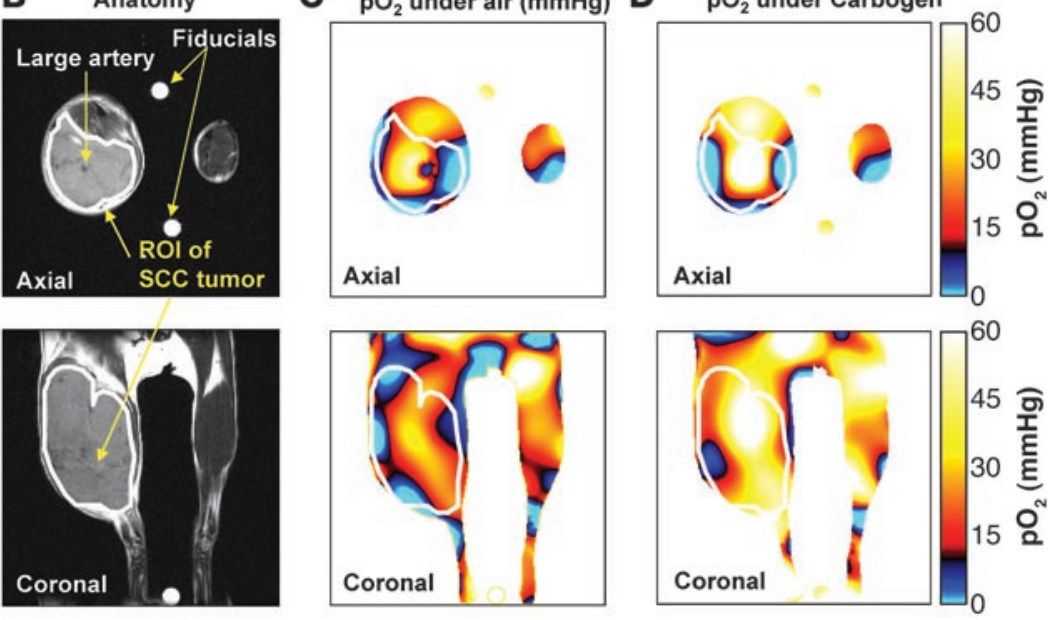

E

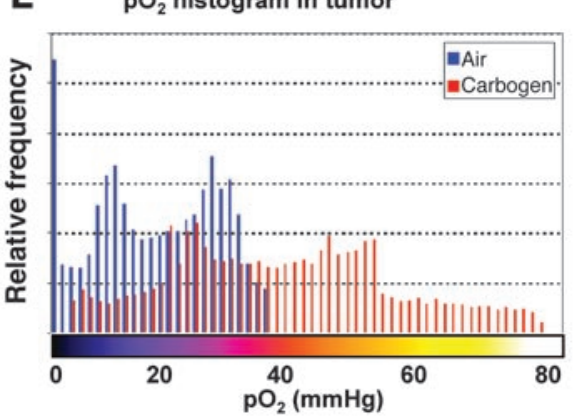

Figure 3

In vivo oxygen mapping of SCC tumor-bearing and normal mouse legs, and visualization of the effect of carbogen breathing on tumor $\mathrm{pO}_{2}$. (A) Cartoon showing the positioning of the tumor-bearing and normal legs along with the fiducials in the resonator for combined EPRI and MRI. (B) $T_{2}$-weighted anatomical images (axial and coronal), in which tumor region could be clearly discriminated. Bright small circles are positional markers (fiducials) for coregistration of EPRI and MRI. (C) $\mathrm{pO}_{2}$ images (axial and coronal) when breathing medical air. (D) $\mathrm{pO}_{2}$ images (axial and coronal) 30 minutes after changing to breathing carbogen. (E) Histograms of $\mathrm{pO}_{2}$ in the tumor region of the same mouse breathing medical air (blue) and carbogen (red). A net increase in the median $\mathrm{pO}_{2}$ was noted upon carbogen breathing. (F) Blood volume image of coronal slice, in which a large blood vessel running vertically can be seen in the center of the tumor. Blood volume was calculated from a difference in image intensity before and after USPIO injection, therefore image intensity from positional markers was canceled. White lines indicate ROI. volume, significant hypoxia exists; (c) carbogen breathing resulted in significant $\mathrm{pO}_{2}$ of hypoxic regions in the tumor using the vascular network. When the $\mathrm{pO}_{2}$ images from EPRI were converted into a frequency histogram (Figure $3 \mathrm{E}$ ), it could be seen that the median $\mathrm{pO}_{2}$ value increased from $16.3 \pm 10.0 \mathrm{mmHg}$ while the mouse was breathing air to $35.5 \pm 17.4 \mathrm{mmHg}$ while the mouse was breathing carbogen. Percentage of the hypoxic fractions that were less than $10 \mathrm{mmHg}$ in tumor region also decreased from $35.1 \%$ to $7.2 \%$ by carbogen breathing. Each 3D EPR image required 8 minutes for acquisition with a spatial resolution of $1.8 \mathrm{~mm}$. The ability to distinguish $\mathrm{pO}_{2}$ with an error limit of $\pm 3-4 \mathrm{mmHg}$ should make it possible to unambiguously monitor changes in response to interventions and pharmacologic challenges.

The capability of overlaying such information with MRI provides the ability to study the relationship between $\mathrm{pO}_{2}$ and other physiological properties such as blood volume and blood flow, which can be routinely obtained by MRI. Results shown in Figure 4 are typical examples of such capabilities, all obtained from the same animal. The axial and coronal images of $\mathrm{pO}_{2}$ distribution from EPRI are shown in Figure 4D. These images show regions of hypoxic cores both in the axial and coronal perspectives. Subsequent to the EPRI data collection, MRI studies were carried out to obtain anatomical information (Figure 4A). In addition to anatomic information from MRI, angiograms, which visualize the major blood vessels in the ROI (Figure 4B), ADC maps (Figure 4F) $(32,33)$, which are indicators of cellularity, and blood flow obtained by arterial spin labeling $(34,35)$ (Figure 4G), were collected. After these scans, USPIO suspension was administered through the tail vein cannula to assess the blood volume (Figure 4, C and E). The images shown in Figure 4, obtained by various modalities in MRI along with the $\mathrm{pO}_{2}$ maps (Figure 4D), provide a more comprehensive assessment of the tumor physiology with respect to the relationship between perfusion-related parameters and the corresponding $\mathrm{pO}_{2}$. In the $\mathrm{pO}_{2}$ maps from EPRI, the hypoxic cores identified from both axial and coronal slices are spatially coincident and are consistent with relatively low levels of blood flow as studied by arterial spin labeling (Figure 4G) and lack of major blood vessels (Figure 4B). The blood volume image represents the microvessel density in the SCC tumor (Figure 4, C and E). The image data suggest that while lower blood volume correlates with decreased $\mathrm{pO}_{2}$, higher blood volume does not necessarily translate into increased $\mathrm{pO}_{2}$. An $\mathrm{ADC}$ value gives quantitative insight into the diffusion of water in a tissue microenvironment in the direction of the diffusion sensitizing gradient. Since major 
A

Anatomy

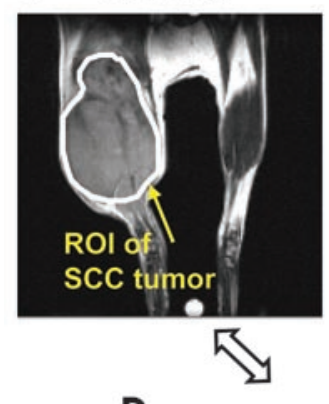

D

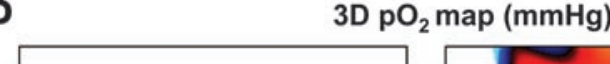

B Angiogram
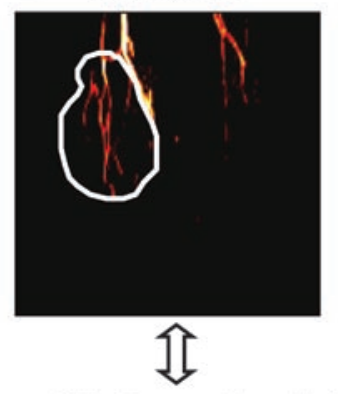

C Blood volume

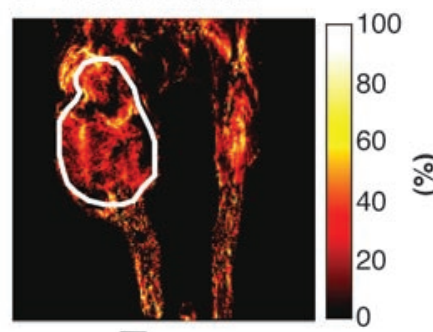

『

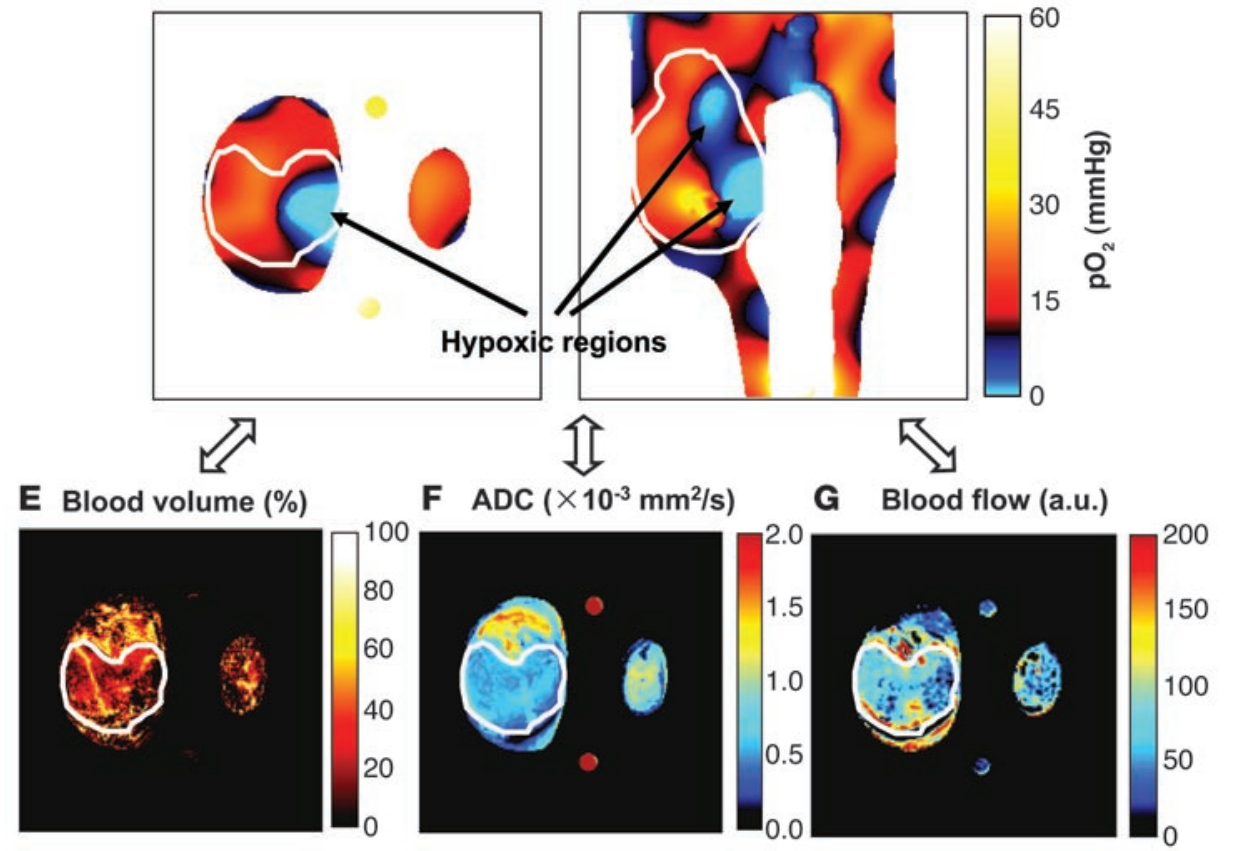

\section{Figure 4}

Fusion of $\mathrm{pO}_{2}$ image derived from EPRI and other flow-related image data from MRI. (A) MRI T2-weighted anatomical image showing the selected ROI of the SCC tumor. (B) $\mathrm{MRI}$ angiogram with an outline of the tumor region derived from MRI $\mathrm{T}_{2}$-weighted anatomical image. (C) MRI coronal blood volume image. (D) Axial and coronal $\mathrm{pO}_{2}$ maps by EPRI. (E) MRI axial blood volume image. (F) MRI ADC image (axial). (G) MRI blood flow image obtained using arterial spin labeling technique. limiting factors of water diffusion are cell membrane and nuclear membrane, ADC values are sensitive to inherent characteristics such as tumor cellularity, ratio of intracellular and extracellular water content, polar character of the cells, and existence of necrosis $(32,33)$. The ADC map (Figure 4F) shows the clearly limited water diffusion within the tumor compared with surrounding normal tissue. The distribution of ADC values was quite homogeneous over the whole tumor region in contrast with $\mathrm{pO}_{2}$ distribution, which is heterogeneous as with blood flow levels. Radiationinduced necrosis causes an increase in ADC, where changes can be observed much earlier than changes of tumor size, making it a possible early surrogate marker of tumor treatment response (32, 33). Implementation of oxygen mapping coregistered with MRI maps, such as blood volume and blood flow obtained before and after treatment, will help oxygen-dependent efficacy of radiation therapy to be understood. Scatter plots of $\mathrm{pO}_{2}$ against blood volume and blood flow in the ROI of the tumor region are shown in Figure 5, A and $\mathrm{B}$, and averaged $\mathrm{pO}_{2}$ values of 4 individual tumorbearing mice were calculated in the different fractions of blood volume (Figure 5C) and blood flow (Figure 5D). The $\mathrm{pO}_{2}$ value obtained by pulsed EPRI positively but not linearly depended on the tumor vascularity, where tumor hypoxia clearly existed only in the region with weak blood perfusion. It is also of interest to note that in regions exhibiting high blood volume and blood flow levels, tumors were relatively better oxygenated.

Most of present treatment strategies and diagnostic tools for cancer are based on characteristic phenotypes commonly observed in many human cancer cell lines. One common phenotype of tumor is aerobic glycolysis, also known as "Warburg effect" (3, 4, 36). Tumor cells meet their energy (ATP) needs by predominantly metabolizing glucose directly to lactate, even in the presence of adequate oxygen, unlike normal tissue that produces most of its ATP by metabolizing glucose to carbon dioxide and water in mitochondria in an oxygen-dependent pathway. Figure $6 \mathrm{~A}$ shows the 3 different ROIs $\left(2.5 \times 2.5 \times 2.5 \mathrm{~mm}^{3}\right.$ cube) for MRS, locations which were selected based on the $\mathrm{pO}_{2}$ status (Figure 6B), and the blood volume levels from MRI (Figure 6C). Typical metabolite peaks such as lactate, creatine, and total choline were distinctly visible in the MRS spectra obtained from the selected ROIs (Figure 6D). The tumor region with higher $\mathrm{pO}_{2}(22.8 \mathrm{mmHg})$ contained clearly high levels of lactate, indicating the predominance of aerobic glycolytic process in normoxic tumor regions. The averaged lactate peak area in radiobiologically oxygenated region (>10 $\mathrm{mmHg}$ ) was significantly higher than that in hypoxic region $(<10 \mathrm{mmHg})$ as shown in Figure $6 \mathrm{E}$. The difference in lactate content can be explained by the limited blood supply and nutrient supply, i.e., limited glucose sup- 

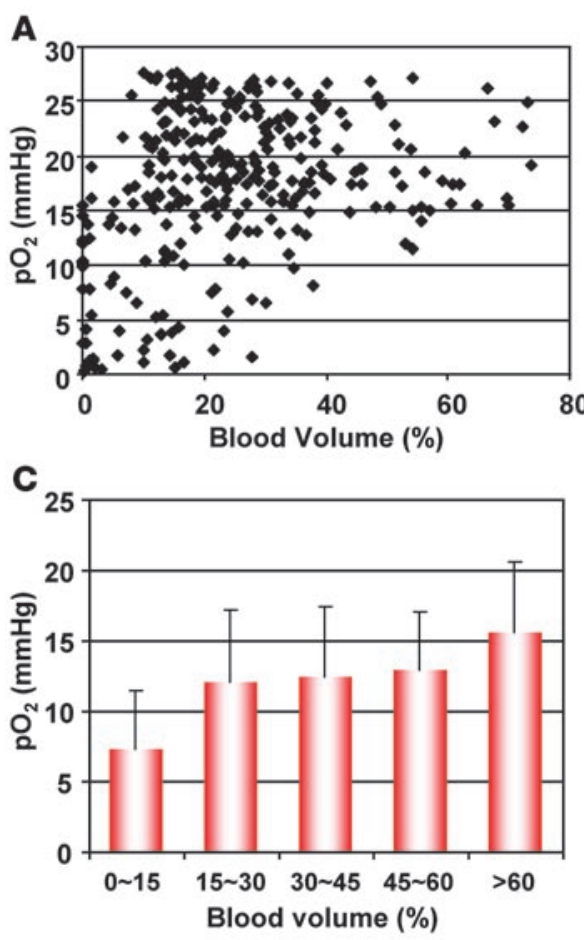
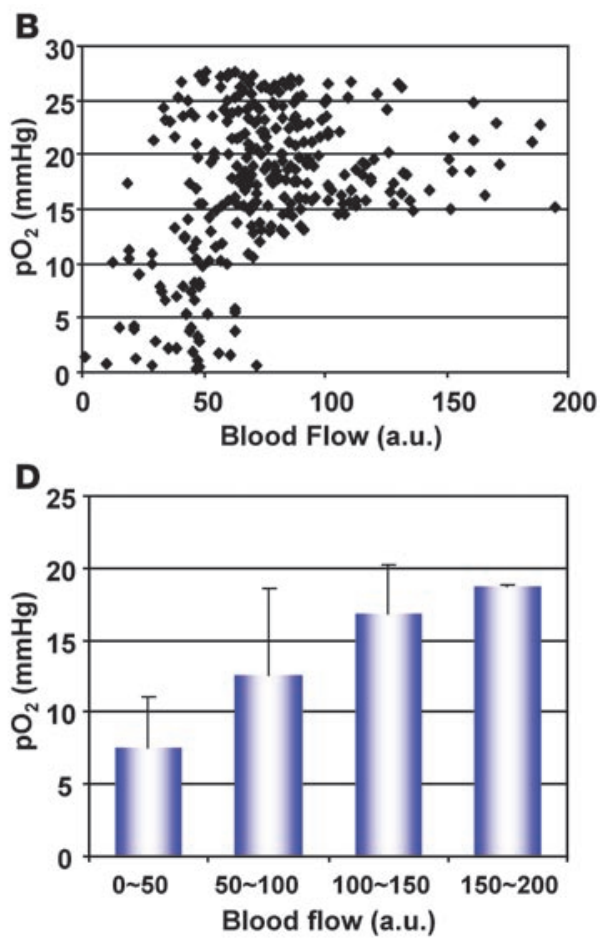

\section{Figure 5}

Voxel-based analysis of the correlation between $\mathrm{pO}_{2}$ status by EPRI and perfusion-related parameters by MRI. The $\mathrm{pO}_{2}$ values from the EPRI in the ROI of SCC tumor region were plotted against the blood volume $(\mathbf{A})$ and blood flow (B) values from the corresponding voxel in $\mathrm{MRI}$ images. The voxel-based $\mathrm{pO}_{2}$ values obtained from 4 different tumor-bearing mice were averaged in the different fractions of blood volume (C) and blood flow values (D). The data were expressed as mean \pm standard deviation. ply in hypoxic regions estimated from the blood volume differences in these regions. The studies described in Figures 2-6, validated by standard phantoms in Figure 1, suggest that valuable physiological information from $\mathrm{pO}_{2}$ maps can be satisfactorily provided by pulsed EPR oxygen imaging technique, which we developed for the first time. The oxygen status can be coregistered with a host of related physiologic and metabolic information derived from MRI/ MRS to gain more complete understanding of tumor physiology.

\section{Discussion}

This study developed a methodology using pulsed EPRI for quantitative determination of tissue $\mathrm{pO}_{2}$ distribution with sensitivity, especially in the radiobiologically hypoxic regions of tumors. Implementation of pulsed EPR-based technique in this study, combined with the recent availability of TAM probes with long lasting effective proton transverse relaxation time $\left(\mathrm{T}_{2} *\right)$ made such a capability feasible (29). Pulsed EPRI-based images from defined phantom objects with oxygen sensitive EPR tracer TAM, equilibrated under different levels of $\mathrm{pO}_{2}$, were used to extract $\mathrm{pO}_{2}$ values by monitoring the oxygendependent shortening of $\mathrm{T}_{2}$ * of TAM. The resultant oxygen maps show that it is possible to monitor changes in $\mathrm{pO}_{2}$ of $\pm 3 \mathrm{mmHg}$. This capability makes it possible to distinguish radiobiologically hypoxic regions in tumors on an absolute $\mathrm{pO}_{2}$ basis, a capability unique to EPRI. Furthermore, since the RF is common between EPRI at $10 \mathrm{mT}$ and MRI at $7 \mathrm{~T}$, a common resonator tuned at $300 \mathrm{MHz}$ can be used for both EPRI and MRI. This provided a useful capability for $\mathrm{pO}_{2}$ images to be reliably overlaid with anatomic and other physiologic information from MRI such as blood volume, blood flow perfusion, etc., without removing the object from the resonator. It is therefore possible to understand the relationship between absolute tissue $\mathrm{pO}_{2}$ levels with perfusion-related parameters.

The $\mathrm{PO}_{2}$ maps from EPRI in the tumor show a heterogeneous distribution in $\mathrm{pO}_{2}$ level. There were regions that are relatively well oxygenated as well as significantly hypoxic regions. Interestingly, upon inhalation of carbogen, significant increases in $\mathrm{pO}_{2}$ were noted around major blood vessel recognized in anatomic and perfusionrelated images. Such regions may represent regions of transiently hypoxic tissue (37). Areas distal from the major blood vessel did not display any increases in $\mathrm{pO}_{2}$, which may result from the presence of longitudinal $\mathrm{pO}_{2}$ gradients (10). Where the vascular oxygen content remains low, the radial diffusion distance of oxygen becomes shorter. Sorg et al. demonstrated, using a window chamber model, that hyperoxic gases have an impact on the tissue $\mathrm{pO}_{2}$ nearer the afferent feeding vessels and yet have little effect on the downstream vasculature (38). Dunn et al. reported the vasoconstrictive effect of carbogen in a site-dependent manner, resulting in decrease of tumor blood flow (39), which may be also a reason for lack of improvement in $\mathrm{pO}_{2}$ by carbogen breathing. The $\mathrm{pO}_{2}$ images obtained from mice breathing air and carbogen can be converted into frequency histograms similar to data obtained by electrode or optical probes, which do not provide spatial information of $\mathrm{pO}_{2}$ but a general distribution from the sampled regions. The median $\mathrm{pO}_{2}$ value in tumor while breathing carbogen was significantly higher than that while breathing air, consistent with earlier results (30).

Prior information for spatial distribution of hypoxia in tumors has the promising potential to improve the efficacy of intensitymodulated radiation therapy (IMRT), which modulates the intensity of the incoming beam of radiation from multiple directions and can be used to achieve a higher degree of spatial agreement of the resulting dose distribution with the target tumor. Generally, in IMRT, the dose distribution is adjusted to have high doses of radiation over the primary target region and low doses that go through particularly critical structures around the tumor. In a few preliminary studies, the $\mathrm{PO}_{2}$ information-based inverse IMRT planning has been said to improve the treatment efficiency over uniform radiation (40-42), but its benefits are still unclear and it needs more investigation. In previous studies, the $\mathrm{pO}_{2}$ information came from invasive Eppendorf electrode or MRI images under 

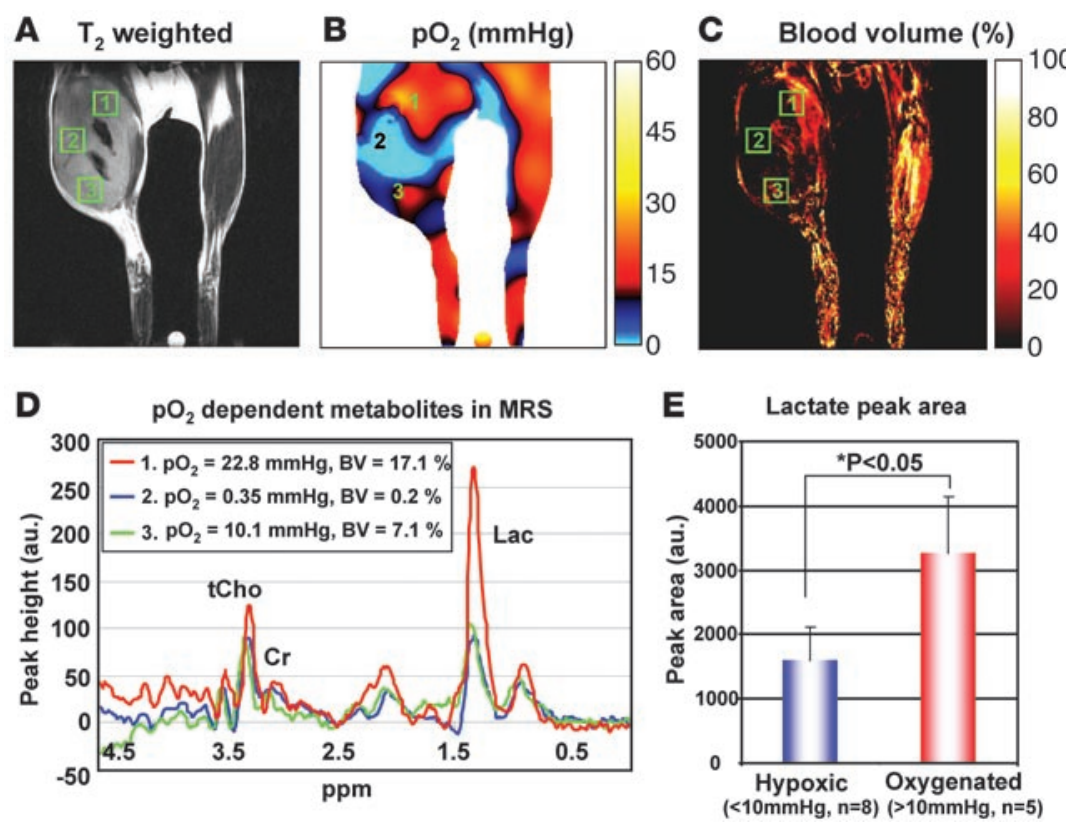

\section{Figure 6}

Images of $\mathrm{pO}_{2}$ distribution and metabolite levels in tumors. (A) $T_{2}$-weighted anatomical MRI image of SCC tumor-bearing mouse and ROI locations for MRS. (B) EPRI $\mathrm{pO}_{2}$ map of the same animal and the corresponding ROIs chosen for MRS. (C) Blood volume image of the slice using USPIO and the corresponding ROls for MRS. Numbers $1-3$ in A-C correspond with numbers $1-3$ in D. (D) Representative MRS spectra obtained from 3 different tumor regions selected with different $\mathrm{pO}_{2}$ and blood volume levels. (E) Averaged lactate peak area of MRS spectra obtained from radiobiologically hypoxic $(<10 \mathrm{mmHg})$ and normoxic $(>10 \mathrm{mmHg})$ regions. High level of lactate production was detected even in the well oxygenated tumor region. BV, blood volume; $\mathrm{Cr}$, creatine; Lac, lactate; tCho, total choline. the assumption that the concentration of MRI contrast media such as Gd-DTPA in the tumor is related to the oxygen tension $(41,42)$. Absolute and rapid $3 \mathrm{D}$ oxygen mapping by pulsed EPRI techniques can help verify the usefulness of $\mathrm{pO}_{2}$-based IMRT.

Recently, transient improvement in tumor $\mathrm{pO}_{2}$ by antiangiogenesis drugs has been getting increasing attention to improve radiotherapy efficacy (43). A combination of $\mathrm{pO}_{2}$ maps obtained by EPRI and perfusion-weighted images by MRI will contribute to a better understanding of tumor oxygen status and its relationship with other blood flow and metabolic profiles, making it possible for a more detailed planning of combination therapy of anticancer drugs and radiation.

The shift in energy production from oxidative phosphorylation to glycolysis in tumor confers great opportunity for diagnosing cancer with positron-emission tomography using ${ }^{18} \mathrm{~F}$-fluorodeoxyglucose. Since lactate is an end product of glycolysis and rarely observed in normal tissue as a strong peak, it provides a reliable MRS marker to distinguish the existence of tumor from normal tissue. Interestingly, in clinical MRS there is considerable debate as to whether the lactate is the end product of the nonoxidative glycolysis and therefore whether its presence can or cannot be correlated with poor $\mathrm{pO}_{2}$ levels or hypoxia, which may exist in tumor $(44,45)$. The combination of EPR oxygen imaging and MRS in this study clearly and noninvasively shows that tumor produces lactate even in the well oxygenated region. Therefore, noninvasive tools for oxygen imaging are helpful for precise understanding of tumor metabolism as demonstrated in this work.

In conclusion, a rapid, noninvasive method to obtain quantitative maps of $\mathrm{pO}_{2}$ in tumor region, coregistered with anatomy, with precision needed to identify radiobiologically hypoxic regions as well as transient hypoxia versus chronic hypoxia, is presented. This method provides such capabilities and therefore may represent a potentially clinically useful modality, which can aid in treatment planning of solid tumors either by radiotherapy alone or in conjunction with chemotherapy. In addition, this method may also be useful for better understanding the relationship between $\mathrm{pO}_{2}$ status and tumor-specific phenotypes such as aerobic glycolysis.

\section{Methods}

Chemicals. The triarylmethyl (TAM) probes oxo63 and oxo31 were obtained from GE Healthcare. We tested the probes and found both oxo63 and oxo31 can be used for EPRI oximetry. They are based on a triarylmethyl (trityl radical) structure, which is derivatized extensively to confer optimal chemical, pharmacological, and EPR characteristics such as stability, water solubility, low toxicity, long in vivo half-lives, single narrow line resonance, and $\mathrm{pO}_{2}$-dependent EPR line widths (23). The dose of TAM used for imaging $(1.125 \mathrm{mmol} / \mathrm{kg})$ was well below the maximally tolerated dose of $2.5-7.0 \mathrm{mmol} / \mathrm{kg}$ and the $\mathrm{LD}_{50}$ of $8 \mathrm{mmol} / \mathrm{kg}(30)$. The pharmacologic half-life of TAM in kidney and blood was 17-21 minutes. The intrinsic line width of TAM is $40 \mathrm{mG}$. The actual line widths (FWHM) at $300 \mathrm{MHz}$ are $180 \mathrm{mG}$ and $133 \mathrm{mG}$, respectively, for oxo63 and oxo31, due to the presence of unresolved hyperfine coupling from ${ }^{13} \mathrm{C}$ and distant protons. USPIO solution Molday ION was purchased from BioPAL Inc. (colloidal size of $30 \mathrm{~ns}, 10 \mathrm{~g} / \mathrm{l} \mathrm{Fe}$ concentration). Other materials used were of analytical grade.

Pulsed (time domain) EPRI and oximetry. Technical details of the pulsed EPR scanner operating at $300 \mathrm{MHz}$, data acquisition based on the singlepoint imaging (SPI) modality, image reconstruction, and the oximetry calculation procedure were described in the earlier reports $(25,29,46)$. Briefly, the SPI is a pure phase-encoding imaging technique. The free induction decays (FIDs) following the excitation pulse ( $80 \mathrm{~ns}, 80 \mathrm{~W}$ ) were sampled using a fast analog digital converter (200 mega samples/s). The repetition time (TR) of $5.5 \mu$ s corresponded to 180,000 excitations and acquisitions per second using $70^{\circ}$ flip angle, satisfying the Ernst angle condition for steady state magnetization. The FIDs were collected under a nested looping of the $x, y, z$ gradients and each time point in the FID underwent phase modulation enabling 3D spatial encoding. Since FIDs last for a couple of microseconds, 1 could generate a sequence of $\mathrm{T}_{2}{ }^{*}$-weighted images, which allowed pixel-wise estimation of in vivo $\mathrm{pO}_{2}$. In order to get reproducible values of $\mathrm{T}_{2}{ }^{*}$ and to retain a more or less uniform image resolution, a set of 3 gradients were used, and data for corresponding images were collected in an interleaved fashion (25). The spatial resolution of SPI modality, in which all 3 directions were phase encoded, employed here does not, in principle, depend on the line width (FWHM) of the TAM probe. If the power spectrum of the pulse is uniform throughout the k-space (image data represented in Fourier space called k-space) for all the gradients used, 
the spatial resolution is simply defined by the FOV and the number of $\mathrm{k}$-samples (elements in $\mathrm{k}$-space). In our measurements, the spatial resolution was $1.8 \mathrm{~mm}$, although the pixel resolution was digitally enhanced in order to coregister with MRI images.

Identical RF coil and fiducial for pulsed EPRI and $7 T M R I$. The basic description of parallel coil resonator used as an identical coil for pulsed EPR and $7 \mathrm{~T}$ MRI operating at $300 \mathrm{MHz}$ was described in an earlier report (47). The quality factor ( $Q$ value) of the coil has to be small in pulsed EPR in order to shorten the receiver recovery time, i.e., dead time, and the $Q$ value in the range 20 30 would be optimal for mouse imaging at $300 \mathrm{MHz}$ in our EPR system, though higher $Q$ is better to obtain the good signal to noise ratio in MRI. Switching of $Q$ values of the coil was done by isolating the damping resistance from the main circuit (48). A parallel coil resonator $(25 \mathrm{~mm}$ i.d. with $25 \mathrm{~mm}$ long) with $Q$ switch was constructed for sequential EPR and NMR imaging.

The use of the position makers (fiducials), which can be seen in both imaging modalities is a straightforward approach to adjust the registration of EPR and NMR images. The position markers were made of 3 capillaries (i.d. $=1 \mathrm{~mm}$ ) containing $3 \mathrm{mM}$ TAM solution, in which TAM could be seen in EPRI and water proton of solvent could be detected by MRI. Two capillaries were placed vertically and the third was placed horizontally. Coregistration of EPR and MRI images was accomplished using code written in MATLAB (Mathworks).

MRI/MRS scanner and pulse sequence. MRI and MRS measurements were done with a $7 \mathrm{~T}$ scanner controlled with ParaVision 3.0.2 (Bruker BioSpin MRI GmbH). After a quick assessment of the sample position by a fast low-angle shot (FLASH) tripilot sequence, $\mathrm{T}_{2}$-weighted anatomical images were obtained using a fast spin echo sequence (RARE) with an echo time (TE) of $13 \mathrm{~ms}$, TR of 2,500 ms, 16 slices, RARE factor 8, resolution of $0.125 \times 0.25 \mathrm{~mm}$, and acquisition time of $80 \mathrm{~s}$. For convenience of coregistration with EPRI, all MRI images had the same FOV of $3.2 \mathrm{~cm}$ and slice thickness $2 \mathrm{~mm}$ unless otherwise mentioned. For $\mathrm{T}_{2}$ mapping, spin echo images were obtained using a multi-slice multi-echo (MSME) sequence, with a 10-echo train and a TE of $15 \mathrm{~ms}$, which required 9 minutes. Diffusion-weighted spin echo images were obtained with TE of $40 \mathrm{~ms}$, TR of 3,000 ms, 8 slices, b-values (diffusion gradient encoding values) of 1.4 and $1044.8 \mathrm{~s} / \mathrm{mm}^{2}$, and resolution of $0.25 \times 0.25 \mathrm{~mm}$. ADC was separately acquired with 3 different diffusion gradient directions (read, phase, and slice), and the average was used as ADC value. Total acquisition time for diffusion-weighted imaging was approximately 20 minutes. To create an angiogram, 64 slices of axial image with $0.5-\mathrm{mm}$ thickness were obtained using a spoiled gradient echo (SPGR) sequence, with very short TR of $20 \mathrm{~ms}$. In such condition, blood protons existing in the slice region are almost saturated and only blood coming freshly into the slice region can give the signal. All 64 axial slices were combined to create data set of 3D angiogram, and coronal slice through the center of tumor was extracted from $3 \mathrm{D}$ angiogram. In order to obtain blood flow image, a single $1 \mathrm{~mm}$ axial slice was chosen through the center of tumor. Relative blood flow was measured using arterial spin labeling (49). A labeling pulse of $2 \mathrm{~s}$ with power of $-6 \mathrm{~dB}$ of $90^{\circ}$ pulse under the field gradient $80 \mathrm{mG} / \mathrm{cm}$ was used at $1.2 \mathrm{~cm}$ rostral to the tumor center to label arterial spins. MRI parameters were as follows: matrix, $128 \times 80$; FOV, $32 \mathrm{~mm}$; TE, $6.8 \mathrm{~ms}$; and TR, 2,000 ms. Total time for the experiment was about 9 minutes. Blood volume calculation was based on the $\mathrm{T}_{2}{ }^{*}$ shortening effect and resulting signal loss by USPIO injection. SPGR sequence images were as follows: matrix, $256 \times 256$; TE, $5.4 \mathrm{~ms}$; TR, $250 \mathrm{~ms}$; slice thickness, $2 \mathrm{~mm}$; scan time, 4 minutes were obtained before and 5 minutes after USPIO injection ( $1.2 \mu \mathrm{l} / \mathrm{g}$ body weight). Percentage of tumor blood volume was estimated by the expression $100 \times\left(S^{\text {pre }}-S^{\text {post }}\right)$ $/\left[\right.$ Spre $\left.^{\text {prost }}\left(W_{b} / W_{t}-1\right)\right]$, where Spre and Spost were the signal intensities of each voxel before and after USPIO injection and $W_{b}$ and $W_{t}$ were intra and extra vascular water fractions.

MRS was performed using a PRESS sequence (1 ms Hermite pulses) to localize a $2.5 \times 2.5 \times 2.5 \mathrm{~mm}^{3}$ voxel positioned either on the tumor or contra-lateral normal tissue. Voxels were chosen to be in regions with minimum fat. A spectral width of 11 PPM over 4,096 data points with a TR/TE of 2,000/21.3 ms were chosen for spectroscopy. An outer volume suppression ( $8 \mathrm{~mm}$ thick; $2 \mathrm{~ms}$ hyperbolic secant pulses [sech]; band width, $10.125 \mathrm{KHz}$ ) was employed to further improve the MRS within the voxel. After uniformity of the magnetic field experienced within a chosen voxel was improved using localized optimization techniques, a single spectrum of the water was acquired. The time domain data was processed without appodization and the line width at half height was measured; this value was used to access the efficiency of field optimization within the voxel across animals. Field optimizations were repeated for each chosen voxel. Water suppression was achieved with the VAPOR (50) sequence (bandwidth $145 \mathrm{~Hz}$; 900/1,800 = 37.24/23.58 ms Hermite pulses, 512 acquisitions, total scan time, $\sim 17 \mathrm{~min}$ ) and the Spectra of the water suppressed, tumor metabolites were obtained after appodization (line broadening, $3 \mathrm{~Hz}$ ).

Experimental design. Female C3H Hen MTV mice were supplied by the Frederick Cancer Research Center, Animal Production. The animals were received at 6 weeks of age and housed 5 per cage in a climate-controlled, circadian rhythm-adjusted room and were allowed food and water ad libitum. SCC cells (SCCVII) were implanted in the femoral muscle of the right hind leg 9 days before the experiments. The tumor size during experimentation was approximately $1.2 \mathrm{~cm}$. Body weight measured before the experiments was $22-28 \mathrm{~g}$. Mice were anesthetized by isoflurane ( $4 \%$ for induction and $1 \%-2 \%$ for maintaining anesthesia) in medical air $(700 \mathrm{ml} / \mathrm{min})$ and mounted prone on a special holder. A pressure transducer (SA Instruments Inc.) was placed on the mouse to monitor and maintain a steady breathing rate at $60 \pm 10$ per minute. During EPR and MRI measurements, core body temperature of the mouse was monitored using a nonmagnetic rectal temperature probe (FISO) and maintained at $37 \pm 1^{\circ} \mathrm{C}$ with flow of warm air. A 30-gauge needle was cannulated into the tail vein and extended using polyethylene tubing (PE-10) for administration of TAM and USPIO solutions. Experiments were carried out in compliance with the Guide for the care and use of laboratory animal resources (National Research Council, 1996) and approved by the National Cancer Institute Animal Care and Use Committee.

Statistics. Differences in $\mathrm{pO}_{2}$ levels and MRS peak areas were compared using the paired or unpaired 2-tailed Student's $t$ test. Differences were considered significant when $P<0.05$. All error bars in the figures were expressed as standard deviations.

\section{Acknowledgments}

We thank Frank Harrington for assembling the gantry for sequential EPRI/MRI studies and the staff of the NIH Mouse Imaging Facility for conducting the studies. This work was supported by the Intramural Research Program, Center for Cancer Research, National Cancer Institute, NIH.

Received for publication January 3, 2008, and accepted in revised form March 5, 2008.

Address correspondence to: Murali C. Krishna, Radiation Biology Branch, Center for Cancer Research, National Cancer Institute, Building 10, Room B3B69, NIH, 9000 Rockville Pike, Bethesda, Maryland 20892-1002, USA. Phone: (301) 496-7511; Fax: (301) 480-2238; E-mail: murali@helix.nih.gov. 
1. Dang, C.V., and Semenza, G.L. 1999. Oncogenic alterations of metabolism. Trends Biochem. Sci. 24:68-72.

2. Helmlinger, G., Yuan, F., Dellian, M., and Jain, R.K. 1997. Interstitial $\mathrm{pH}$ and $\mathrm{pO} 2$ gradients in solid tumors in vivo: high-resolution measurements reveal a lack of correlation. Nat. Med. 3:177-182.

3. Gatenby, R.A., and Gillies, R.J. 2004. Why do cancers have high aerobic glycolysis? Nat. Rev. Cancer. 4:891-899.

4. Kim, J.W., and Dang, C.V. 2006. Cancer's molecular sweet tooth and the Warburg effect. Cancer Res. 66:8927-8930

5. Semenza, G.L., et al. 2001. 'The metabolism of tumours': 70 years later. Novartis Found. Symp. 240:251-260; discussion 260-254.

6. Harris, A.L. 2002. Hypoxia - a key regulatory factor in tumour growth. Nat. Rev. Cancer. 2:38-47.

7. Erickson, K., et al. 2003. Effect of longitudinal oxygen gradients on effectiveness of manipulation of tumor oxygenation. Cancer Res. 63:4705-4712.

8. Lanzen, J., et al. 2006. Direct demonstration of instabilities in oxygen concentrations within the extravascular compartment of an experimental tumor. Cancer Res. 66:2219-2223.

9. Dewhirst, M.W., et al. 2007. Multiple etiologies of tumor hypoxia require multifaceted solutions. Clin. Cancer Res. 13:375-377.

10. Dewhirst, M.W., et al. 1999. Quantification of longitudinal tissue $\mathrm{pO} 2$ gradients in window chamber tumours: impact on tumour hypoxia. Br. J. Cancer. 79:1717-1722.

11. Hockel, M., et al. 1996. Association between tumor hypoxia and malignant progression in advanced cancer of the uterine cervix. Cancer Res. 56:4509-4515.

12. Hockel, M., and Vaupel, P. 2001. Tumor hypoxia: definitions and current clinical, biologic, and molecular aspects. J. Natl. Cancer Inst. 93:266-276.

13. Brown, J.M., and Giaccia, A.J. 1998. The unique physiology of solid tumors: opportunities (and problems) for cancer therapy. Cancer Res. 58:1408-1416

14. Vaupel, P., Schlenger, K., Knoop, C., and Hockel, M. 1991. Oxygenation of human tumors: evaluation of tissue oxygen distribution in breast cancers by computerized O2 tension measurements. Cancer Res. 51:3316-3322.

15. Vaupel, P., Hockel, M., and Mayer, A. 2007. Detection and characterization of tumor hypoxia using $\mathrm{pO} 2$ histography. Antioxid. Redox Signal. 9:1221-1235.

16. Tatum, J.L., et al. 2006. Hypoxia: importance in tumor biology, noninvasive measurement by imaging, and value of its measurement in the management of cancer therapy. Int. J. Radiat. Biol. 82:699-757.

17. Hockel, M., Schlenger, K., Knoop, C., and Vaupel, P. 1991. Oxygenation of carcinomas of the uterine cervix: evaluation by computerized $\mathrm{O} 2$ tension measurements. Cancer Res. 51:6098-6102.

18. Hockel, M., et al. 1993. Intratumoral pO2 predicts survival in advanced cancer of the uterine cervix. Radiother. Oncol. 26:45-50.

19. Brown, J.M., and Le, Q.T. 2002. Tumor hypoxia is important in radiotherapy, but how should we measure it? Int. J. Radiat. Oncol. Biol. Phys. 54:1299-1301.

20. Rasey, J.S., Hofstrand, P.D., Chin, L.K., and Tewson, T.J. 1999. Characterization of [18F]fluoroetanidazole, a new radiopharmaceutical for detecting tumor hypoxia. J. Nucl. Med. 40:1072-1079

21. Rajendran, J.G., et al. 2006. Tumor hypoxia imaging with [F-18] fluoromisonidazole positron emission tomography in head and neck cancer. Clin. Cancer Res. 12:5435-5441.

22. Evans, S.M., et al. 2006. EF5 binding and clinical outcome in human soft tissue sarcomas. Int. J. Radiat. Oncol. Biol. Phys. 64:922-927.

23. Ardenkjaer-Larsen, J.H., et al. 1998. EPR and DNP properties of certain novel single electron contrast agents intended for oximetric imaging. J. Magn. Reson. 133:1-12.

24. Elas, M., et al. 2003. Quantitative tumor oxymetric images from 4D electron paramagnetic resonance imaging (EPRI): methodology and comparison with blood oxygen level-dependent (BOLD) MRI. Magn. Reson. Med. 49:682-691.

25. Matsumoto, K., et al. 2006. Electron paramagnetic resonance imaging of tumor hypoxia: enhanced spatial and temporal resolution for in vivo $\mathrm{pO} 2$ determination. Magn. Reson. Med. 55:1157-1163.

26. Elas, M., et al. 2006. Electron paramagnetic resonance oxygen images correlate spatially and quantitatively with Oxylite oxygen measurements. Clin. Cancer Res. 12:4209-4217.

27. Lai, C.S., Hopwood, L.E., Hyde, J.S., and Lukiewicz, S. 1982. ESR studies of O2 uptake by Chinese hamster ovary cells during the cell cycle. Proc. Natl. Acad. Sci. U. S. A. 79:1166-1170.

28. Murugesan, R., et al. 1997. In vivo imaging of a stable paramagnetic probe by pulsed-radiofrequency electron paramagnetic resonance spectroscopy. Magn. Reson. Med. 38:409-414.

29. Devasahayam, N., et al. 2007. Strategies for improved temporal and spectral resolution in in vivo oximetric imaging using time-domain EPR Magn. Reson. Med. 57:776-783.

30. Krishna, M.C., et al. 2002. Overhauser enhanced magnetic resonance imaging for tumor oximetry: coregistration of tumor anatomy and tissue oxygen concentration. Proc. Natl. Acad. Sci. U. S. A 99:2216-2221.

31. Anzai, Y., et al. 1997. MR angiography with an ultrasmall superparamagnetic iron oxide blood pool agent. J. Magn. Reson. Imaging. 7:209-214.

32. Chenevert, T.L., et al. 2000. Diffusion magnetic resonance imaging: an early surrogate marker of therapeutic efficacy in brain tumors. J. Natl. Cancer Inst. 92:2029-2036

33. Moffat, B.A., et al. 2005. Functional diffusion map: a noninvasive MRI biomarker for early stratification of clinical brain tumor response. Proc. Natl. Acad. Sci. U. S. A. 102:5524-5529.

34. Williams, D.S., Detre, J.A., Leigh, J.S., and Koretsky, A.P. 1992. Magnetic resonance imaging of perfusion using spin inversion of arterial water. Proc. Natl. Acad. Sci. U. S. A. 89:212-216.
35. Barbier, E.L., Silva, A.C., Kim, S.G., and Koretsky, A.P. 2001. Perfusion imaging using dynamic arterial spin labeling (DASL). Magn. Reson. Med. 45:1021-1029.

36. Pedersen, P.L. 2007. Warburg, me and Hexokinase 2: multiple discoveries of key molecular events underlying one of cancers' most common phenotypes, the "Warburg Effect", i.e., elevated glycolysis in the presence of oxygen. J. Bioenerg. Biomembr. 39:211-222.

37. Dewhirst, M.W. 1998. Concepts of oxygen transport at the microcirculatory level. Semin. Radiat. Oncol. 8:143-150.

38. Sorg, B.S., Moeller, B.J., Donovan, O., Cao, Y., and Dewhirst, M.W. 2005. Hyperspectral imaging of hemoglobin saturation in tumor microvasculature and tumor hypoxia development. J. Biomed. Opt. 10:44004.

39. Dunn, T.J., et al. 1999. The effects of hyperoxic and hypercarbic gases on tumour blood flow. Br.J. Cancer. 80:117-126.

40. Chao, K.S., et al. 2001. A novel approach to overcome hypoxic tumor resistance: Cu-ATSM-guided intensity-modulated radiation therapy. Int. J. Radiat. Oncol. Biol. Phys. 49:1171-1182.

41. Malinen, E., Sovik, A., Hristov, D., Bruland, O.S., and Olsen, D.R. 2006. Adapting radiotherapy to hypoxic tumours. Phys. Med. Biol. 51:4903-4921.

42. Sovik, A., et al. 2007. Radiotherapy adapted to spatial and temporal variability in tumor hypoxia. Int. J. Radiat. Oncol. Biol. Phys. 68:1496-1504.

43. Ansiaux, R., et al. 2006. Mechanism of reoxygenation after antiangiogenic therapy using SU5416 and its importance for guiding combined antitumor therapy. Cancer Res. 66:9698-9704.

44. Star-Lack, J.M., et al. 2000. In vivo 1 H MR spectroscopy of human head and neck lymph node metastasis and comparison with oxygen tension measurements. AJNR Am. J. Neuroradiol. 21:183-193.

45. Li, X., et al. 2005. Relationship of MR-derived lactate, mobile lipids, and relative blood volume for gliomas in vivo. AJNR Am. J. Neuroradiol. 26:760-769.

46. Subramanian, S., et al. 2002. Single-point (constant-time) imaging in radiofrequency Fourier transform electron paramagnetic resonance. Magn. Reson. Med. 48:370-379.

47. Devasahayam, N., et al. 2000. Parallel coil resonators for time-domain radiofrequency electron paramagnetic resonance imaging of biological objects. J. Magn. Reson. 142:168-176.

48. Yamada, K., et al. 2002. Evaluation and comparison of pulsed and continuous wave radiofrequency electron paramagnetic resonance techniques for in vivo detection and imaging of free radicals. J. Magn. Reson. 154:287-297.

49. Zhang, W., Williams, D.S., and Koretsky, A.P. 1993. Measurement of rat brain perfusion by NMR using spin labeling of arterial water: in vivo determination of the degree of spin labeling. Magn. Reson. Med. 29:416-421.

50. Tkac, I., Starcuk, Z., Choi, I.Y., and Gruetter, R. 1999. In vivo $1 \mathrm{H}$ NMR spectroscopy of rat brain at 1 ms echo time. Magn. Reson. Med. 41:649-656. 